\title{
Variables psicológicas positivas en pacientes con cáncer
}

\author{
Cerezo Guzmán, $M^{a}$ Victoria
}

Doctora por la Universidad de Málaga. Departamento de Psicología y Metodología de la Investigación en las Ciencias del Comportamiento.

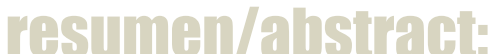

La psicología positiva está emergiendo en la investigación actual y en este artículo se pretende hacer un recorrido por algunas de las variables contempladas desde esta perspectiva: bienestar, emociones positivas, inteligencia emocional, optimismo, resiliencia y autoestima. Se ha destacado la relación existente entre cada una de dichas variables en una población cada vez más numerosa: los pacientes oncológicos. Los resultados de las distintas investigaciones convergen en los beneficios de explorar y fomentar la salud positiva a través de intervenciones psicológicas específicas, de forma que se les pueda ayudar a mejorar aspectos relevantes para su salud tales como su estado de ánimo, incrementar la calidad de vida, disminuir el distrés emocional, o facilitar estrategias de afrontamiento más adaptativas a los pacientes oncológicos.

Positive psychology is currently emerging in research. This is the reason why it has been checked a group of variables considered in this perspective: wellbeing, positive emotions, emotional intelligence, optimism, resilience and self-esteem. It has been highlighted the relationship between each of these variables in an increasingly large population: cancer patients. The results of the various investigations converge on the benefits of exploring and promoting positive health through specific psychological interventions, so that they can help to improve health relevant aspects such as mood, increase quality

of life, reduce emotional distress, or facilitate more adaptive coping strategies for cancer patients.

\section{palabras clave/keywords:}

psicología positiva, pacientes oncológicos, optimismo, inteligencia emocional positive psychology, cancer patients, optimism, emotional intelligence

\section{Introducción}

La psicología positiva es una rama de la psicología de reciente aparición que busca comprender, a través de la investigación científica, los procesos que subyacen a las cualidades y emociones positivas del ser humano. El estudio de la patología y la debilidad del ser humano ha sido el foco casi distintivo durante muchos años de la psicología, sesgando el estudio de la mente humana a su parte más patogénica y olvidando por tanto, que el ser humano también tiene características positivas como el optimismo, la creatividad, el sentido del humor o la ilusión, entre muchas otras cualidades y fortalezas, que le ayudan a adaptarse a cada situación y convertirse en un ser con posibilidades y éxitos (Seligman y 
Csikszentmihalyi, 2000). Se pueden encontrar antecedentes de la psicología positiva en filósofos como Aristóteles (que dedicó parte de sus escritos a la eudaimonia, término griego habitualmente traducido como felicidad), pero también en psicólogos como Abraham Maslow o Carl Rogers, pertenecientes a la corriente de la psicología humanista. El objetivo de la psicología positiva es conseguir que las personas podamos valorar las experiencias pasadas como satisfactorias, manteniendo la esperanza y el optimismo hacia el futuro, mientras en el presente apreciamos la felicidad a la vez que nos dejamos fluir (flow) (Peterson, Park y Seligman, 2006; Seligman y Csikszentmihalyi, 2000).

Los investigadores dedicados a la prevención en el ámbito de la salud descubrieron que hay fortalezas humanas que actúan como amortiguadores contra la enfermedad mental: el coraje, el optimismo, las habilidades interpersonales, la fe, la esperanza, la honestidad, la perseverancia, la autoconsciencia y la capacidad de fluir, por nombrar algunos (Peterson et al., 2006). Y centrándonos en la salud, desde este enfoque psicológico también se vislumbra de la mano de Seligman (2008) un nuevo término: salud positiva, paralelo a la psicología positiva, que se describe como un estado más allá de la mera ausencia de enfermedad y que puede practicarse. De ser así, lograr el bienestar, evitar el sufrimiento, tener propósitos, relaciones y emociones positivas, ser optimistas, etc., serían nuestras mejores armas para evitar un trastorno mental (Hou, Law y Fu, 2010; Peterson et al., 2006; Pressman y Cohen, 2005). Según Seligman (2008), la salud mental se asienta en emociones positivas, compromiso, propósito, relaciones positivas y logros positivos, siendo algo más que la ausencia de enferme- dad mental. La salud mental, además de ser cuantificable, predice la ausencia de depresión, produce mayor rendimiento y mejor salud física. Por tanto, tener salud física o mental positiva ("salud positiva") nos protege de enfermedades, gracias al fuerte vínculo existente entre la psicología positiva (bienestar, optimismo y otras emociones positivas) y el estado de salud. Diversas investigaciones han concluido que las personas con salud positiva viven más, producen menos gastos en salud; $o$ en caso de tener una enfermedad, tienen un mejor pronóstico (Cohen, Alper, Doyle, Treanor y Turner, 2006; Cohen y Pressman, 2006; Kubzhansky y Thurston, 2007; Seligman, 2008).

Algunas de las variables más estudiadas dentro de la psicología positiva con pacientes oncológicos a tratar en este artículo son: el bienestar, la inteligencia emocional, el optimismo, la resiliencia o la autoestima.

\section{Bienestar y cáncer}

Conocer en profundidad las bases que sustentan el bienestar es un objetivo de gran importancia, no solo porque puede permitir que cada vez haya personas más satisfechas consigo mismas, sino que también las emociones positivas y la felicidad están asociadas a múltiples beneficios: mejor salud, mejores relaciones sociales, mejor rendimiento laboral, incluso mayor tendencia a beneficiar a otros (Lyubomirsky, King y Diener, 2005). En psicología se suele considerar el bienestar como un sinónimo de felicidad, con el que podemos valorar los aspectos cognitivos y afectivos de manera más amplia (Diener, 2009; Diener, Emmons, Larsen y Griffin, 1985; Fierro, 2006; Vázquez y Hervás, 2008). Se podría decir que el bienestar de una persona sería la valoración positiva de su vida, basada tanto en la congruencia entre sus aspiraciones y 
logros alcanzados (componente cognitivo), como en presentar un estado emocional y afectivo óptimo (componente afectivo). Al mismo tiempo, la percepción de ausencia de bienestar, consistiría en una valoración negativa de la vida, producto de la incongruencia entre expectativas y logros, así como el predominio de emociones y afectos displacenteros (Fierro, 2006, 2008).

De entre los factores personales que podamos contemplar, nada afecta tanto a la vida feliz como la salud física. Eso no obsta a que personas con poca salud física, o seriamente afectadas por una discapacidad, muestren una vitalidad envidiable, como por ejemplo, el conocido físico Stephen Hawking. Si la salud física refleja nuestro nivel de bienestar o felicidad, ni que decir tiene que la salud mental también se ve muy condicionada a ésta (Fierro, 2009).

Los estudios que tratan del bienestar y la felicidad en cáncer suelen estar relacionados con intervenciones psicológicas con las que se pretende conseguir mejorar el estado de bienestar de los participantes, desde los distintos enfoques, técnicas, o variables estudiadas (Cameron, Booth, Schlatter, Ziginskas y Harman, 2007; Cerezo, OrtizTallo y Cardenal, 2009 a,c; Font y Rodríguez, 2007; Iwamitsu et al., 2005). En los estudios con pacientes oncológicos se ha encontrado, de manera global, que la felicidad se relaciona positivamente con una adecuada reparación emocional (Cerezo y Ortiz-Tallo, 2007) y mejor calidad de vida (Carver et al., 2005; Novoa, Vargas, Obispo, Pertuz y Rivera, 2010; GarcíaViniegras y González, 2007), destacando la necesidad de que las estrategias de atención sanitaria a los pacientes con cáncer incluyan elementos clínico-psicológicos que promuevan una mejoría del bienestar psicológico de estos pacientes.

\section{Emociones positivas y cáncer}

Por otro lado, se sabe que las emociones positivas generan adaptabilidad, pueden prevenir la aparición de enfermedades (el resfriado común o la gripe) y complicaciones en enfermedades más graves (en los accidentes cerebrovasculares o las tasas de mortalidad en los ancianos); además, pueden reducir el dolor, incrementando su umbral debido al efecto analgésico de la liberación de opiáceos endógenos asociados a las emociones positivas (Cohen et al., 2006; Cohen y Pressman, 2006; Fredrickson, 1998; Pressman y Cohen, 2005; Seligman, 2008), demostrando también su efecto positivo en las personas con cáncer (Bozo, Gündogdu y Büyükasik-Çolak, 2009; Hou et al., 2010). Experimentar emociones positivas tales como la alegría, entusiasmo, orgullo, satisfacción, gratitud, esperanza, admiración, humor, etc., lleva a estados mentales y modos de comportamiento que de forma indirecta preparan al individuo para enfrentar con éxito las dificultades y adversidades venideras (Fredrickson, 2003; Hou et al., 2010). Las emociones positivas pueden ser el medio para solventar muchos de los problemas que generan las emociones negativas, nos sirven para conseguir sobreponernos a momentos difíciles y salir fortalecidos de ellos; pueden guiarnos hacia la prevención, el tratamiento y el afrontamiento, de forma que se transforman en verdaderas armas para afrontar problemas. La alegría, el amor, el orgullo, la complacencia, etc., aunque sean fenomenológicamente distintas entre sí, comparten muchas propiedades, ya que mejoran el pensamiento y la acción, fomentan el desarrollo y crecimiento personal, mantienen y mejoran los recursos físicos, intelectuales, psicológicos y sociales disponibles para momen- 
tos futuros de crisis o adversidad, generan habilidades psicológicas que permiten descubrir y crear posibilidades, y desarrollan habilidades sociales adecuadas para generar vínculos entre personas y aprendizaje de conductas de ayuda (Vecina, 2006). Y en cuanto a los pacientes de cáncer, se demuestra que los afectos positivos les aportan beneficios teniendo mejores hábitos de consumo y de autocuidado, mejor calidad y cantidad de sueño y practicando más ejercicio físico (Lyubomirsky et al., 2005; Vázquez y Castilla, 2007).

\section{Inteligencia emocional y cáncer}

Otra variable a considerar desde la psicología positiva es la inteligencia emocional: habilidad para procesar la información, para percibir, asimilar, comprender y regular las propias emociones y las de los demás, promoviendo el crecimiento emocional e intelectual (Mayer y Salovey, 1997). Se ha observado que la inteligencia emocional correlaciona positivamente con liderazgo y con autoestima, así como negativamente con ansiedad, se asocia a mayor capacidad de respuesta a estimulación afectiva negativa, así como a una recuperación de la misma más rápida (Smith et al., 2011). Esto indica que niveles adecuados de inteligencia emocional se asocian a menor deterioro psicológico causado por acontecimientos vitales estresantes o experiencias traumáticas, es decir, que la inteligencia emocional modera significativamente el impacto del estresor (Mikolajczak, Petrides, Coumans y Luminet, 2009). El metaanálisis que relaciona inteligencia emocional y salud muestra que la inteligencia emocional es un fuerte predictor de buena salud, siendo más evidente esta relación en las mujeres (Martins, Ramalho y Morin, 2010). En cuanto a los estudios con pacientes oncológicos, esa asociación se corrobora, ya que también es un fuerte predictor de la adaptación psicológica en pacientes diagnosticados de cáncer. Concretamente, los pacientes con cáncer urológico con adecuada inteligencia emocional mostraron menores estados de ansiedad, menos preocupación y mayor apoyo social percibido (Smith et al., 2011). En pacientes con cáncer de mama, la inteligencia emocional se relaciona con bienestar personal, por tanto, debe trabajarse eficientemente para que se convierta en una capacidad estable (Cerezo et al., 2007, 2009 a,b,c).

\section{Optimismo y cáncer}

Dentro de las variables psicológicas positivas también se encuadra el optimismo. El optimismo es una característica disposicional de personalidad que media entre los acontecimientos externos y la interpretación personal que hacemos de ellos, es la tendencia a esperar que el futuro nos depare resultados favorables, el valor que nos ayuda a enfrentar las dificultades con buen ánimo y perseverancia, descubriendo lo positivo de las personas y las circunstancias, y que nos hace confiar en nuestras capacidades y posibilidades junto con la ayuda que podamos recibir (Avia y Vázquez, 1998; Scheier y Carver, 1993). Las diferencias entre las personas optimistas y pesimistas tienen que ver con la forma de relacionarse con el mundo y la importancia o impacto que esto tiene en sus vidas. Estas personas difieren en la forma de afrontar los problemas y adversidades, difieren en sus recursos individuales, sociales y económicos, incluso en el riesgo de presentar alguna psicopatología (Carver, Scheier y Segerstrom, 2010). Diversos estudios indican que las personas optimistas tienden a abordar mejor las situaciones difíciles con es- 
trategias de afrontamiento más adaptativas, con lo que parecen tener más resistencia a los efectos psicológicos y biológicos de los eventos estresantes, controlando mejor el estrés y/o las enfermedades, manejando mejor eventos potencialmente traumáticos y mostrando mayor perseverancia, mayor salud física, bienestar y teniendo más logros vitales (Bozo et al., 2009; Carver, Scheier, Miller y Fulford, 2009; Nes y Segerstrom, 2006; Rasmussen, Scheier y Greenhouse, 2009).

Casi todos los estudios que versan sobre el optimismo, incluidos los metaanálisis más recientes, informan de su valor predictivo sobre la salud y el bienestar, además de actuar como modulador sobre los eventos estresantes, paliando el sufrimiento y el malestar de aquellos que sufren, tienen estrés o enfermedades graves, incluso puede actuar como potenciador del bienestar y la salud en aquellas personas que, sin presentar trastornos, pretenden mejorar su calidad de vida (Rasmussen et al., 2009; Seligman, 2002).

Se han estudiado las relaciones entre optimismo y muy diferentes enfermedades, demostrando por ejemplo, una fuerte relación entre altos niveles de optimismo y la vitalidad emocional con una mayor protección y mejor pronóstico para las enfermedades cardiovasculares, comparándose al mismo tiempo con los efectos de la depresión, que aumenta significativamente el riesgo de accidentes o problemas cardíacos (Kubzansky y Thurston, 2007; Seligman, 2008). El optimismo también se asocia a una mejor respuesta inmunocompetente, comparada con la de personas pesimistas (Seligman y Csikszentmihalyi, 2000); alarga la vida y mejora el estado de salud de los ancianos
(Cohen et al., 2006; Cohen y Pressman, 2006), incluso en personas con problemas de fertilidad u otro tipo de decepciones intensas de la vida, los optimistas lo asumen con menos síntomas depresivos o ansiosos que los pesimistas (Carver et al., 2010). En enfermedades como el cáncer, se ha observado que las personas optimistas parecen tener una mejor supervivencia y respuesta inmune (Allison, Guichard, Fung y Gilain, 2003; Aspinwall y Tedeschi, 2010a; Carver et al., 2005), así como que muestran menores niveles de ansiedad durante los tratamientos, y mejor calidad de vida previa y posterior al diagnóstico (Carver et al., 2010; Lee, Cohen, Edgar, Laizner y Gagnon, 2006). Esto indica que una mayor tendencia al optimismo se suele relacionar con estrategias de afrontamiento activas y con el desarrollo de conductas de solución de problemas y autocuidado, incluso cuando hablamos de cáncer, donde se ha comprobado, por ejemplo, que las pacientes optimistas tienen mejor ajuste psicológico tras el diagnóstico de cáncer de mama y utilizan mejores estrategias de afrontamiento, presentan mejor actividad inmunológica, estado de ánimo y apoyo percibido, y les disminuye el dolor (Vázquez y Castilla, 2007); aún más, se puede confirmar que el optimismo predice un nivel óptimo de bienestar, varios años después (Carver et al., 2005). Lo que se puede intuir de estos estudios es que un paciente optimista tiende más probablemente a poner en práctica hábitos que mejoren su salud, a mantener buenas relaciones sociales en las que percibir apoyo, así como a que sus estados afectivos positivos tengan un efecto fisiológico directo que le ayuden a retardar el curso de su enfermedad. 


\section{Resiliencia y cáncer}

La resiliencia, entendida como la capacidad del ser humano de resistir y rehacerse frente a los embates de la vida, la capacidad de recuperarse y mantener una conducta adaptativa después del desastre, surge como parte de la psicología positiva suscitando investigaciones que se preguntan mediante qué mecanismos algunas personas consiguen aprender de sus experiencias e incluso encontrar beneficios en ellas. Sin despreciar el horror y la gravedad de esas experiencias, hay que destacar que es en esas situaciones extremas donde el ser humano tiene la oportunidad darse cuenta de sus propias capacidades latentes, de volver a construir su forma de entender el mundo y su sistema de valores, tiene la oportunidad de replantearse su manera de vivir y de modificar sus creencias, de manera que en esta reconstrucción puede darse, y de hecho se da en muchos casos, un aprendizaje y un crecimiento personal (Vera, 2008). En la definición de resiliencia se incluyen cualidades como la inteligencia, la salud, el temperamento, darle sentido a las experiencias, tener habilidades para identificar y relacionarse con modelos positivos, tener buena voluntad y confianza en las relaciones, tener habilidades de solución de problemas, flexibilidad o perseverancia (Becoña, 2006; Carver, 1998). La resiliencia fomenta la salud mental y parece una realidad confirmada por el testimonio de muchísimas personas que, aun habiendo vivido una situación traumática, han conseguido encajarla y seguir desenvolviéndose $\mathrm{y}$ viviendo, incluso, en un nivel superior, como si el trauma vivido y asumido hubiese desarrollado en ellos recursos latentes e insospechados (Bowen, Morasca y Meischke, 2003; Vázquez y Castilla, 2007).
En cáncer, esta resiliencia se ha venido llamando crecimiento postraumático, ya que las emociones y cogniciones positivas pueden ser también resultado de la vivencia de situaciones traumáticas. El crecimiento postraumático se definiría como la capacidad de las personas de generar respuestas de crecimiento o maduración personal sin negar la vivencia de la adversidad vivida, pero dándole un significado o sentido a la misma (Costa y Gil, 2008; Vázquez y Castilla, 2007). El evento traumático induce una crisis existencial que desencadena un proceso de "rumiación constructiva", asociado a la búsqueda de significado en medio de una gran incertidumbre y percepción de vulnerabilidad. Es un proceso reparador, una reestructuración cognitiva que ayuda a encontrar una explicación a lo ocurrido (Calhoum y Tedeschi, 2006). La respuesta de crecimiento puede entenderse como el resultado del proceso de atribuir significado a un evento estresante (Park, 2004). Se relaciona mucho con el optimismo, al que consideran como parte del proceso de crecimiento.

La mayoría de los estudios psicooncológicos se centran en las mujeres con cáncer de mama y concluyen que el ajuste psicológico es mucho más adecuado cuando las mujeres le encuentran sentido a la vivencia (Bellizzi y Blank, 2006; Scrignaro, Barni y Magrin, 2010), tras los tratamientos tienen menos consecuencias emocionales negativas (Aspinwall y MacNamara 2005; Aydin, 2008; Bozo et al., 2009; Salsman, Segerstrom, Brechting, Carlson y Andrykowski, 2009), se cuidan más durante y una vez acabados los tratamientos, llevando una vida más saludable, comprometiéndose más fuertemente con su salud (Vázquez y Castilla, 2007); y suelen ser las mujeres de 
mayor edad a las que les es más fácil considerarse resilientes ante las adversidades en comparación con las mujeres más jóvenes (Bennett et al., 2008; Morse y Adams-Price, 2008). En estudios en los que se trabaja terapéuticamente para ayudar a mejorar el crecimiento postraumático, se han encontrado importantes cambios positivos en el grupo de participantes, sobre todo en la mejora de síntomas de estrés postraumático, con lo que se comprueba que las intervenciones o tratamientos en este ámbito son favorables (Hart, Wilson y Hittner, 2006; Knaevelsrud, Liedl y Maercker, 2010).

\section{Autoestima y cáncer}

La última variable a considerar en este artículo es la autoestima. La autoestima es un conjunto de actitudes que dependen de las percepciones, pensamientos, evaluaciones, sentimientos y tendencias de comportamiento dirigidas hacia nosotros mismos, hacia nuestra forma de comportarnos, hacia nuestro cuerpo y nuestro carácter. En resumen, es la percepción evaluativa de uno mismo. La importancia que tiene la autoestima radica en que nos concierne por completo: a nuestra manera de ser y al sentido de nuestra valía personal; además de que funciona como motor de nuestra vida: habla de nosotros, de cómo nos percibimos y comportamos y de lo que esperamos del otro. Es uno de los factores más relevantes para el bienestar personal, clave para relacionarnos con nuestro entorno de una forma satisfactoria. Por lo tanto, no puede menos que afectar a nuestra manera de estar y actuar en el mundo y de relacionarnos con los demás. Nada en nuestra manera de pensar, de sentir, de decidir y de desenvolvernos escapa a la influencia de la autoestima (Bonet, 1997). La autoestima adecuada, por tanto, comprende cubrir las necesida- des de respeto por uno mismo con sentimientos como confianza, competencia, logros, maestría, independencia y libertad.

Los pacientes con cáncer suelen ver su autoestima deteriorada en algún momento o circunstancia del proceso de superación de la enfermedad como consecuencia de los tratamientos, de sus efectos secundarios, de los cambios en su imagen corporal, de la incertidumbre ante el futuro, etc. En la sociedad actual, se nos exige ser perfectos: altos, guapos, inteligentes, resolutivos, responsables, con buen físico... y cuando a una persona le diagnostican una enfermedad como puede ser el cáncer, la autoestima se tambalea por la abismal incongruencia entre lo que socialmente se espera de ella y lo que le deparará la enfermedad de aquí en adelante. El cáncer y los tratamientos oncológicos recibidos pueden dañar la autoestima de los pacientes en diferentes sentidos: por los cambios en la imagen corporal (alopecia, aumento de peso, cicatrices, secuelas de la cirugía, en especial, la mastectomía, etc.), por los cambios en su independencia y estilo de vida (no poder trabajar o desempeñar las tareas habituales, no poder realizar deporte, etc.), o por las consecuencias derivadas de los tratamientos sobre algún órgano o función determinada (de la fertilidad, el habla, la movilidad, alteraciones en necesidades básicas, como comer, respirar o defecar, etc.). La presencia de alguna de estas consecuencias les puede hacer sentir menos valioso, menos útil o atractivo, generando sentimientos de inseguridad y provocando aislamiento, menos relaciones sociales, distanciamiento de la pareja y de familiares, y en definitiva, generando trastornos de gravedad variable. El aspecto sexual, también se ve muy alterado por el estado de ánimo vul- 
nerable, así como por las dificultades que se le presentan tras los tratamientos. En mujeres mastectomizadas, se ha estudiado la relación entre la baja autoestima y la alteración de la vida sexual: diferentes estudios ponen de manifiesto que las mujeres mastectomizadas están menos satisfechas con su imagen corporal que las pacientes con cirugía conservadora, aunque estén en proceso de reconstrucción de la mama, con lo que se puede afirmar que la mastectomía impacta negativamente en la autoestima de las pacientes, interfiriendo además en el apoyo percibido, en su vida social y sexual, así como en su bienestar (Brandberg et al., 2008; González, Rodríguez, Alonso, de León y Gutiérrez, 2010; Lepore, Glaser y Roberts, 2008; Markopoulos et al., 2009; Sebastián, Manos, Bueno y Mateos, 2008). Otro grupo de pacientes que también sufre muy especialmente este tipo de dificultades es el de cáncer colorrectal, que además de ver interferida su funcionalidad más básica, alterada su fertilidad y resentida su autoestima, sienten que se altera gravemente su vida sexual, llegando a tener disfunciones sexuales hasta el $57 \%$ de los afectados (da Silva et al., 2008). De nuevo, la mayoría de las investigaciones incluyen tratamientos psicológicos en los que se trabaja con los pacientes de cáncer para mejorar su calidad de vida, su autoestima, su bienestar, etc., concluyendo que es necesario intervenir para ayudarles a adaptarse mejor a su situación oncológica, trabajando diferentes facetas psicológicas: emocionales, sociales, habilidades, etc., en función del tipo de cáncer que presentan (Bartoces et al., 2009; Lee et al., 2006; Sebastián et al., 2008).

\section{Conclusiones}

La literatura científica deja patente los esfuerzos que se están haciendo por contri- buir a que mejore la salud de las personas afectadas de cáncer en cuanto a algunas de las variables más importantes consideradas desde la perspectiva de la psicología positiva. Parece que tener salud física o mental positiva nos protege de enfermedades, gracias al fuerte vínculo existente entre nuestra faceta psicológica positiva (bienestar, optimismo y otras emociones positivas) y el estado de salud (Fierro, 2008; Seligman, 2002, 2008; Seligman y Csikszentmihalyi, 2000; Vera, 2008).

Desde la psicología positiva también hay un gran grupo de científicos trabajando para corroborar los beneficios de esta nueva orientación psicológica, explorando y fomentando la salud positiva, las cualidades y fortalezas de cada persona, y diseñando intervenciones novedosas para aquellos con dificultades en su salud, de forma que se les pueda ayudar a mejorar, con el objetivo de alcanzar la máxima prevención en las enfermedades físicas y mentales (Seligman, 2008).

En enfermedades como el cáncer, la psicología positiva aún tiene mucho camino que recorrer, pero ya se sabe que las personas con más optimismo o fortalezas, por ejemplo, parecen tener mejor salud y mayor bienestar (Aspinwall y Tedeschi, 2010a; Cardenal, Cerezo, Martínez, Ortiz-Tallo y Blanca, 2012; Carver et al., 2005; Carver et al., 2010; Lee et al., 2006; Vázquez y Castilla, 2007). Estos hallazgos tan sugerentes pueden invitar a los investigadores de la psicología de la salud a desarrollar sus trabajos bajo el prisma de la psicología positiva, pudiendo llegar a aportar a las personas con diversas enfermedades una nueva forma de recuperar su salud psicológica y quizás mejorar de algún modo su salud física. 


\section{Referencigs}

Allison, P. J., Guichard, C., Fung, K. y Gilain, L. (2003). Dispositional optimism predicts survival status 1 year after diagnosis in head and neck cancer patients. Journal of Clinical Oncology, 21(3), 543-548.

Aspinwall L. G. y MacNamara A. (2005). Taking positive changes seriously: Toward a positive psychology of cancer survivorship and resilience. Cancer, 104(11), 25492556. DOI 10.1002/cncr.21244

Aspinwall, L. G. y Tedeschi, R. G. (2010a). The Value of Positive Psychology for Health Psychology: Progress and Pitfalls in Examining the Relation of Positive Phenomena to Health. Annals of Behavioral Medicine, 39, 4-15. DOI 10.1007/s12160-009-9153-0

Avia, M. D. y Vázquez, C. (1998). Optimismo inteligente: Psicología de las emociones positivas. Madrid: Alianza.

Aydin, E. (2008). Trauma and Resilience in Women Diagnosed with Breast Cancer: A Transactional Analysis Perspective. Transactional Analysis Journal, 38(4), 323135.

Bartoces, M. G., Severson, R. K., Rusin, B. A., Schwartz, K. L., Ruterbusch, J. J. y Neale, A. V. (2009). Quality of Life and Self-Esteem of Long-Term Survivors of Invasive and Noninvasive Cervical Cancer. Journal of Women's Health, 18(5), 655-661.

Becoña, E. (2006). Resiliencia: definición, características y utilidad del concepto. Revista de Psicopatología y Psicología Clínica, 11, 125-146.

Bellizzi, K. M. y Blank, T. 0. (2006). Predicting Posttraumatic Growth in Breast Cancer Survivors. Health Psychology, 25(1), 47-56. DOI: 10.1037/0278-6133.25.1.47

Bennett, J. A., Cameron, L., Brown, P., Whitehead, L., Porter, T. y Parkes, T. 0. (2008). Resilience in cancer: few older cancer survivors seek information and support services following a cancer diagnosis. The Gerontologist, 48, 140-151.

Bonet, J. V. (1997). Sé amigo de ti mismo: manual de autoestima. Cantabria: Sal Terrae. Maliaño.

Bowen, D. J., Morasca, A. A. y Meischke, H. (2003). Measures and Correlates of Resilience. Women y Health, 38 (2), 65-76.

Bozo, Ö., Gündogdu, E. y Büyükasik-Çolak, C. (2009). The Moderating Role of Different Sources of Perceived Social Support on the Dispositional Optimism-Posttraumatic Growth Relationship in Postoperative Breast Cancer Patients. Journal of Health Psychology, 14, 1009-1020. DOI: $10.1177 / 1359105309342295$
Brandberg, Y., Sandelin, K., Erikson, S., Jurell, G., Liljegren, A., Lindblom, A., Lindén, A., von Wachenfeldt, A., Wickman, M. y Arver, B. (2008). Psychological Reactions, Quality of Life, and Body Image After Bilateral Prophylactic Mastectomy in Women At High Risk for Breast Cancer: A Prospective 1-Year Follow-Up Study. Journal of Clinical Oncology, 26(24), 3943-3949. DOI: 10.1200/ JC0.2007.13.9568

Calhoum, L. G. y Tedeschi, R. G. (2006). The Foundations of Posttraumatic Growth: an expanded framework. En: Calhoum, L. G., y Tedeschi, R. G., editors. Handbook of Posttraumatic Growth (pp. 3-23). Mahwah, New York: Lawrence Erlbaum Associate Publishers.

Cameron, L. D., Booth, R. J., Schlatter, M., Ziginskas, D. y Harman, J. E. (2007). Changes in emotion regulation and psychological adjustment following use of a group psychosocial support program for women recently diagnosed with breast cancer. Psycho-Oncology, 16, 171-180. DOI: 10.1002/pon.1050

Cardenal, V., Cerezo, M. V., Martínez, J., Ortiz-Tallo, M. y Blanca, M. J. (2012). Personality, Emotions and Coping Styles: Predictive Value for the Evolution of Cancer $\mathrm{Pa}$ tients. The Spanish Journal of Psychology, 15(2), 756-767.

Carver, C. S. (1998). Resilience and thriving: Issues, models and linkages. Journal of Social Issues, 54, 245-266.

Carver, C. S., Scheier, M. F., Miller, C. J. y Fulford, D. (2009). Optimism. In: Lopez S. J., Snyder C. R., eds. Oxford Handbook of Positive Psychology. $2^{\text {nd }}$ ed. (pp. 303-311). New York: Oxford University Press.

Carver, C. S., Scheier, M. F. y Segerstrom, S. C. (2010). Optimism. Clinical Psychology Review, 30, 879-889. DOI:10.1016/j.cpr.2010.01.006

Carver, C. S., Smith, R. G., Derhagopian, R. P., Antoni, M. H., Petronis, V. M. y Weiss, S. (2005). Optimistic personality and psychosocial well-being during treatment predict psychosocial well-being among long-term survivors of breast cancer. Health Psychology, 24(5), 508-516. DOI: 10.1037/0278-6133.24.5.508

Cerezo M. V. y Ortiz-Tallo, M. (2007). Relación entre Inteligencia Emocional y Bienestar Personal en mujeres operadas de Cáncer de Mama. Poster presentado en las Jornadas "Emociones y Bienestar" de la SEAS (Sociedad Española para el Estudio de la Ansiedad y el Estrés), Septiembre, 27-29, Madrid.

Cerezo, M. V., Ortiz-Tallo, M. y Cardenal, V. (2009a). Expresión de emociones y bienestar en un grupo de mujeres con cáncer de mama: una intervención psicológica. Revista Latinoamericana de Psicología, 41(1), 131-140.

Cerezo, M. V., Ortiz-Tallo, M. y Cardenal, V. (2009b). Estudio longitudinal sobre bienestar y emociones: la reparación emocional y el cáncer de mama. Poster presentado en las II Jornadas de Emociones y Bienestar. 
Sociedad Española de Ansiedad y Estrés. 1-3, 0ctubre, en Madrid, España.

Cerezo, M. V., Ortiz-Tallo, M. y Cardenal, V. (2009c). Programa de Inteligencia Emocional: La Reparación Emocional y el Cáncer de Mama. Conferencia presentada en The II International Emotional Intelligence Congress, 16-18 Septiembre, en Santander, España.

Cohen, S., Alper, C. M., Doyle, W. J., Treanor, J. J. y Turner, R. B. (2006). Positive emotional style predicts resistance to illness after experimental exposure to rhinovirus or Influenza A virus. Psychosomatic Medicine, 68, 809-815.

Cohen, S. y Pressman, S. D. (2006). Positive affect and health. Current Directions in Psychological Science, 15, 122-125.

Costa, G. y Gil, F. L. (2008). Respuesta cognitiva y crecimiento postraumático durante el primer año de diagnóstico del cáncer. Psicooncología, 5(1), 27-37.

da Silva, G. M., Hull, T., Roberts, P. L., Ruiz, D. E., Wexner, S. D., Weiss, E. G., Nogueras, J. J., Daniel, N., Bast, J., Hammel, J. y Sands, D. (2008) The Effect of Colorectal Surgery in Female Sexual Function, Body Image, Self-Esteem and General Health: A Prospective Study. Annals of Surgery, 248(2), 266-272. DOI: 10.1097/SLA.0b013e3181820cf4

Diener, E. D. (2009). (Ed.). Assessing Well-Being. The collected works of Ed Diener. Oxford, UK: Springer.

Diener, E. D., Emmons, R. A., Larsen, R. J. y Griffin, S. (1985). The Satisfaction With Life Scale. Journal of Personality Assessment, 49, 71-75.

Fierro, A. (2006). Bienestar personal, adaptación social y factores de personalidad: Estudios con las Escalas Eudemon. Clínica y Salud, 17(3), 297-318.

Fierro, A. (2008). Conocimiento contra infelicidad: Para una psicología epicúrea. Escritos de Psicología, 2(1), 7-23.

Fierro, A. (2009). El buen vivir: De los genes a la práctica. Paradigma, 23-27.

Font, A. y Rodríguez, E. (2007). Eficacia de las intervenciones psicológicas en mujeres con cáncer de mama. Psicooncología, 4(2-3), 423-446.

Fredrickson, B. L. (1998). What good are positive emotions? Review of General Psychology, 2(3), 300-319.

Fredrickson, B. L. (2003). The value of positive emotions. American Scientist, 91, 330-335.

García-Viniegras, C. R. V. y González, M. (2007). Bienestar psicológico y cáncer de mama. Avances en Psicología Latinoamericana, 25(1), 72-80.

González, M., Rodríguez, M. E., Alonso, M. E., de León, L. y Gutiérrez, J. M. (2010). Mujeres con cáncer de mama y su sexualidad. $11^{\circ}$ Congreso Virtual de Psiquiatría. In- terpsiquis. http://www.bibliopsiquis.com/bibliopsiquis/ handle/10401/1064

Hart, K. E., Wilson, T. L. y Hittner, J. B. (2006). A Psychosocial Resilience Model to Account for Medical Well-being in Relation to Sense of Coherence. Journal of Health Psychology, 11(6), 857-862. DOI: 10.1177/1359105306069082

Hou, W. K., Law, C. C. y Fu, Y. T. (2010). Does change in positive affect mediate and/or moderate the impact of symptom distress on psychological adjustment after cancer diagnosis? A prospective analysis, Psychology y Health, 25(4), 417-431. DOI: 10.1080/08870440802559375

Iwamitsu, Y., Shimoda, K., Abe, H., Tani, T., Okawa, M. y Back, R. (2005). Anxiety, Emotional suppression, and Psychological Distress before and after breast cancer diagnosis. Psychosomatics, 46, 19-24.

Knaevelsrud, C., Liedl, A. y Maercker, A. (2010). Posttraumatic Growth, Optimism and Openness as outcomes of a Cognitive-behavioural Intervention for Posttraumatic stress Reactions. Journal of Health Psychology, 15(7), 1030-1038. DOI: 10.1177/1359105309360073

Kubzhansky, L. D. y Thurston, R. (2007). Emotional vitality and incident coronary heart disease. Archives of General Psychiatry, 64, 1393-1401.

Lee, V., Cohen, R., Edgar, L., Laizner, A. M. y Gagnon, A. J. (2006). Meaning-making intervention during breast or colorectal cancer treatment improves self-esteem, optimism, and self-efficacy. Social Science and Medicine, 62, 3133-3145. DOI:10.1016/.socscimed.2005.11.041

Lepore, S., J., Glaser, D. B. y Roberts, K. J. (2008). On the positive relation between received social support and negative affect: a test of the triage and self-esteem threat models in women with breast cancer. Psycho-Oncology, 17(12), 1210-1215.

Lyubomirsky, S., King, L. y Diener, E. (2005). The Benefits of Frequent Positive Affect: Does Happiness Lead to Success? Psychological Bulletin, 131(6), 803-855.

Markopoulos, C., Tsaroucha, A. K., Kouskos, E., Mantas, D., Antonopoulou, Z. y Karvelis, S. (2009). Impact of Breast Cancer Surgery on the Self-esteem and Sexual Life of Female Patients. The Journal of International Medical Research, 37, 182-188.

Martins, A., Ramalho, N. y Morin, E. (2010). A comprehensive meta-analysis of the relationship between Emotional Intelligence and health. Personality and Individual Differences, 49, 554-564. DOI:10.1016/j.paid.2010.05.029

Mayer, J. D. y Salovey, P. (1997). "What is emotional intelligence?" En P. Salovey y D. Sluyter (eds.), Emotional development and emotional intelligence: implications for educators (pp. 3-31). New York: Basic Books.

Mikolajczak, M., Petrides, K. V., Coumans, N. y Luminet, 0. (2009). The moderating effect of trait emotional intel- 
ligence on mood deterioration following laboratory-induced stress. International Journal of Clinical and Health Psychology, 9, 455-477.

Morse, L. y Adams-Price, C. (2008). Positive psychology and aging: positive outlook in younger and older breast cancer patients. The Gerontologist, 48, 21.

Nes, L. y Segerstrom, S. (2006). Dispositional optimism and coping: A meta-analytic review. Personality and Social Psychology Review, 10(3), 235-251.

Novoa, M. M., Vargas, R. M., Obispo, S. A., Pertuz, M. y Rivera, Y. K. (2010). Evaluación de la calidad de vida y bienestar psicológico en pacientes postquirúrgicos con cáncer de tiroides. International Journal of Psychology and Psychological Therapy, 10(2), 315-329.

Park, C. L. (2004). The Notion of Growth Following Stressful Life Experiences: Problems and Prospects. Psychological Inquiry, 15, 69-76.

Peterson, C., Park, N. y Seligman, M. E. P. (2006). Greater strengths of character and recovery from illness. The Journal of Positive Psychology, 1(1), 17-26. DOl: 10.1080/17439760500372739

Pressman, S. D. y Cohen, S. (2005). Does positive affect influence health? Psychological Bulletin, 131(6), 925-971. DOI: 10.1037/0033-2909.131.6.925

Rasmussen, H. N., Scheier, M. F. y Greenhouse, J. B. (2009). Optimism and physical health: A meta-analytic review. Annals of Behavioral Medicine, 37(3), 239-256.

Salsman, J. M., Segerstrom, S. C., Brechting, E. H., Carlson, C. R. y Andrykowski, M. A. (2009). Posttraumatic growth and PTSD symptomatology among colorectal cancer survivors: a 3-month longitudinal examination of cognitive processing Psychooncology, 18(1), 30-41.

Scheier, M. F. y Carver, C. S. (1993). On the power of positive thinking: the benefits of being optimistic. Psychological Science, 2, 26-30.

Scrignaro, M., Barni, S. y Magrin, M. E. (2010). The combined contribution of social support and coping strategies in predicting post-traumatic growth: a longitudinal study on cancer patients. Psycho-oncology. DOI: 10.1002/ pon.1782

Sebastián, J., Manos, D., Bueno, M. J. y Mateos, N. (2008). Body image and self-esteem in women with breast Cancer participating in a psychosocial Intervention program. Psychology in Spain, 12(1), 13-25.

Seligman, M. E. P. (2002). Authentic Happiness: Using the New Positive Psychology to Realize Your Potential for Lasting Fulfillment. New York: Free Press/Simon and Schuster.

Seligman, M. E. P. (2008). Positive Health. Applied Psychology: An International Review, 57, 3-18. DOI: 10.1111/j.1464-0597.2008.00351.x
Seligman, M. E. P. y Csikszentmihalyi, M. (2000). Positive Psychology: An introduction. American Psychologist, 55(1), 5-14. DOI: 10.1037//0003-066X.55.1.5

Smith, S. G., Turner, B., Pati, J., Petrides, K. V., Sevdalis, N. y Green, J. S. A. (2011). Psychological impairment in patients urgently referred for prostate and bladder cancer investigations: the role of trait emotional intelligence and perceived social support. Support Care Cancer. DOI: 10.1007/s00520-011-1129-5.

Vázquez, C. y Castilla, C. (2007). Emociones positivas y crecimiento postraumático en el cáncer de mama. Psicooncología, 4(2-3), 385-404.

Vázquez, C. y Hervás, C. (2008). Psicología positiva aplicada. Bilbao: Desclee de Brower.

Vecina, M. L. (2006). Emociones Positivas. Papeles del Psicólogo, 27(1), 9-17

Vera, B. (2008). Psicología positiva: una nueva forma de entender la psicología. Madrid: Calamar Ediciones.

Fecha de recepción: 04/06/2013

Fecha de aceptación: 30/10/2013 